\title{
Reemplazo total de rodilla en pacientes con anquilosis. Reporte de tres casos y revisión bibliográfica
}

\author{
Juan Ignacio Pérez Abdala, Ignacio García-Mansilla, Tomás I. Nicolino, Carlos Yacuzzi, Lisandro Carbó, \\ Julián Costantini, Matías Costa Paz \\ Sector de Rodilla, Instituto de Ortopedia y Traumatología "Prof. Dr. Carlos E. Ottolenghi”, Hospital Italiano de Buenos Aires, \\ Ciudad Autónoma de Buenos Aires, Argentina
}

\begin{abstract}
RESUMEN
Introducción: El reemplazo total de rodilla en pacientes con anquilosis representa un desafío para el cirujano, tanto por la alta demanda técnica de la cirugía como por la elevada tasa de complicaciones comunicada. El objetivo de este artículo es presentar tres pacientes con anquilosis tratados con un reemplazo total de rodilla y una revisión bibliográfica actualizada. Materiales y Métodos: Se evaluaron las distintas etiologías, la movilidad preoperatoria, la técnica quirúrgica y el tipo de implante utilizado en cada caso. En el seguimiento, se evaluaron la tasa de complicaciones, el rango de movilidad y la supervivencia del implante. Por último, se realizó una revisión actualizada de la bibliografía. Resultados: Dos pacientes tenían artritis reumatoide juvenil y uno, una secuela de osteomielitis crónica de rodilla. Los pacientes no tenían movilidad articular y sufrían un severo compromiso para realizar las actividades de la vida cotidiana. En los tres pacientes, se efectuó un abordaje pararrotuliano medial seguido de una amplia liberación de partes blandas. En dos casos, se utilizaron prótesis primarias con vástagos y, en el restante, una prótesis de bisagra rotacional. El rango de movilidad alcanzado fue de $90^{\circ}$ en promedio y los pacientes refirieron una tasa alta de satisfacción. No se registraron complicaciones. Conclusiones: El reemplazo total de rodilla en pacientes con anquilosis es una opción terapéutica por considerar, y tiene un impacto beneficioso en la calidad de vida. Por la complejidad que representa debe afrontarse como una cirugía de revisión, con una detenida planificación preoperatoria.
\end{abstract}

Palabras clave: Anquilosis; rodilla; artroplastia.

Nivel de Evidencia: IV

Total Knee Replacement in Patients with Ankylosis. Report of Three Cases and Literature Review

\section{ABSTRACT}

Introduction: Performing a Total Knee Replacement (TKR) in patients with ankylosed knees is technically demanding and associated with considerable complications. The purpose of this study is to report three cases of patients with ankylosed knees treated with TKR and present an updated literature review. Materials and Methods: We evaluated etiologies, preoperative range of motion, surgical technique and type of implant utilized in each case. Complications and postoperative range of motion were also analyzed. Radiographs were used to evaluate loosening or osteolysis. Lastly, we performed an updated literature review. Results: The etiologies were juvenile rheumatoid arthritis in two cases and chronic osteomyelitis in one. The patients did not have range of motion at all and the ability to perform daily life activities was severely affected. A medial parapatellar approach was used in all cases followed by an extensive soft tissue release. A primary posterior-stabilized design was used in two cases and a rotatinghinge in one case. The mean postoperative range of motion was $90^{\circ}$ and all three patients reported a high satisfaction rate. No complications were reported. Conclusions: TKR in patients with ankylosed knees has substantially improved the clinical outcome and the arc of movement. Due to its complexity, it must be approached as a revision surgery, with careful preoperative planning. Keywords: Ankylosis; knee; arthroplasty.

Level of Evidence: IV

Recibido el 30-5-2021. Aceptado luego de la evaluación el 24-6-2021 • Dr. JUAN IGNACIO PÉREZ ABDALA • ignacio.perez@ hospitalitaliano.org.ar ID https://orcid.org/0000-0002-5398-6876 Cómo citar este artículo: Pérez Abdala Jl, García-Mansilla I, Nicolino TI, Yacuzzi C, Carbó L, Costantini J, Costa Paz M. Reemplazo total de rodilla en pacientes con anquilosis. Reporte de tres casos y revisión bibliográfica. Rev Asoc Argent Ortop Traumatol 2021;86(4):519-528. https://doi.org/10.15417/issn. 1852-7434.2021.86.4.1383 


\section{INTRODUCCIÓN}

El reemplazo total de rodilla (RTR) tiene como objetivo mejorar el dolor y la movilidad, otorgar estabilidad y permitir una adecuada calidad de vida, considerando las expectativas de cada paciente. ${ }^{1-3}$ El éxito se basa, en parte, en los resultados funcionales obtenidos, y el rango de movilidad preoperatorio es una variable determinante en el rango posoperatorio esperado..$^{3-5}$ Entre el $1 \%$ y el $13 \%$ de las prótesis primarias, se colocan en pacientes con rigidez de rodilla. ${ }^{3,-8}$ Las etiologías más frecuentes son: artrosis primaria, artritis reumatoide, artrosis postraumática y secuelas de una enfermedad infecciosa. ${ }^{3,69}$ Se distinguen, al menos, dos escenarios en estos pacientes: con un rango de movilidad $<50^{\circ}$ y sin movilidad de la rodilla, estos cuadros se denominan rigidez y anquilosis, respectivamente. ${ }^{3,6,10,11}$ Esta entidad provoca un compromiso severo de la calidad de vida debido a las limitaciones funcionales que genera; por lo tanto, el manejo quirúrgico tiene un papel inexorable. ${ }^{12,13}$

Esta situación plantea un enorme desafío para el cirujano, tanto por la alta demanda de la técnica quirúrgica, como por la elevada tasa de complicaciones publicada. ${ }^{11,12}$ La cirugía debe ser enfocada como una revisión, con una detallada planificación preoperatoria. El abordaje es un punto clave en estos casos, muchas veces, es necesario ampliarlo agregando gestos quirúrgicos, tales como liberación del retináculo lateral, osteotomía de la tuberosidad anterior de la tibia, rectus snip o cuadriceplastia en V-Y., ${ }^{3,611-15}$ Debido a la retracción de partes blandas y sus adherencias, resulta difícil identificar las estructuras ligamentarias, lo que puede provocar complicaciones, como rotura del tendón rotuliano o avulsiones ligamentarias. Por este motivo, con frecuencia, es necesario utilizar diseños de prótesis con mayor constricción.

Las complicaciones posoperatorias son importantes, la tasa publicada llega hasta el $53 \%$ y, en su mayoría, se trata de infecciones y necrosis de la herida. ${ }^{6,8,13,16-18}$ Sin embargo, la calidad de vida de los pacientes mejora considerablemente y refieren altas tasas de satisfacción. ${ }^{6,9,19}$

Debido a la baja incidencia de este cuadro, la bibliografía es escasa y, en gran parte, se trata de series con poca cantidad de pacientes. Los objetivos de este artículo son presentar tres pacientes con anquilosis tratados con RTR y una revisión bibliográfica del tema.

\section{CASOS CLÍNICOS}

\section{Caso 1}

Mujer de 18 años, con artritis reumatoide juvenil y una intervención quirúrgica durante la infancia. Presenta anquilosis de la rodilla derecha en flexión de $100^{\circ}$, sin deformidad significativa en el plano coronal. En la rodilla izquierda, tiene un rango de movilidad de $90^{\circ}$, con una contractura en flexión de $30^{\circ}$. Tiene dificultad para deambular, subir y bajar escaleras y sentarse. El Knee Society Score (KSS) de la rodilla derecha medido antes de la cirugía era 26/50. En las radiografías, se puede observar la anquilosis ósea tanto tibiofemoral como rotulofemoral de la rodilla derecha (Figura 1).

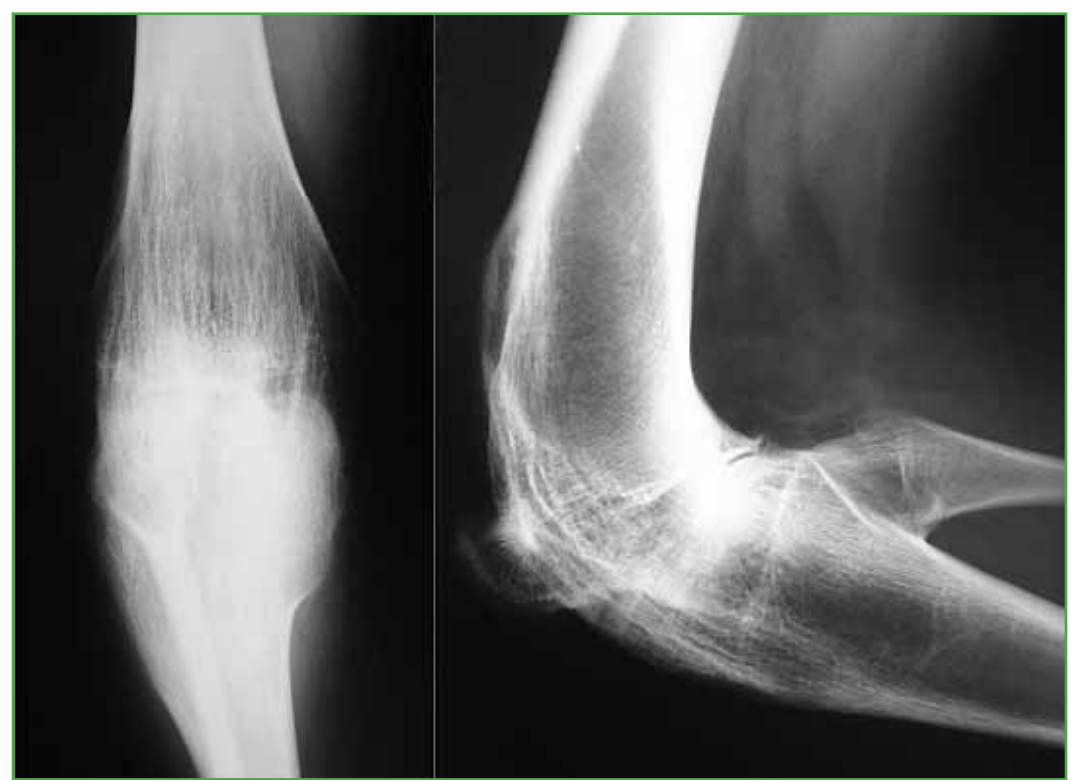

Figura 1. Radiografía preoperatoria de rodilla derecha, de frente y de perfil. 


\section{Técnica quirúrgica}

Se realizó un abordaje anterior de rodilla y artrotomía pararrotuliana medial, seguida de una liberación de adherencias y disección subperióstica a demanda hasta exponer la anquilosis rotulofemoral. Se efectuó una osteotomía, se resecó la grasa de Hoffa necesaria y se luxó la rótula hacia lateral. Se restituyeron goteras y se realizó una osteotomía tibiofemoral. En primer lugar, se practicó el corte tibial con guía extramedular consiguiendo una buena visión posterior para liberar partes blandas, permitiendo llegar a una extensión completa. Se realizaron cortes de fémur con guía endomedular, y se evaluaron las brechas. Se probaron los componentes y se evaluó el encarrilamiento rotuliano, donde en algunas situaciones, es necesaria la liberación lateral. En este caso, se logró un correcto balance articular y una buena estabilidad; por lo tanto, se utilizó una prótesis primaria estabilizada posterior con vástago tibial y femoral.

A los 13 años de seguimiento, la paciente tiene un rango de movilidad de $90^{\circ}$, con una extensión completa (Figura 2).

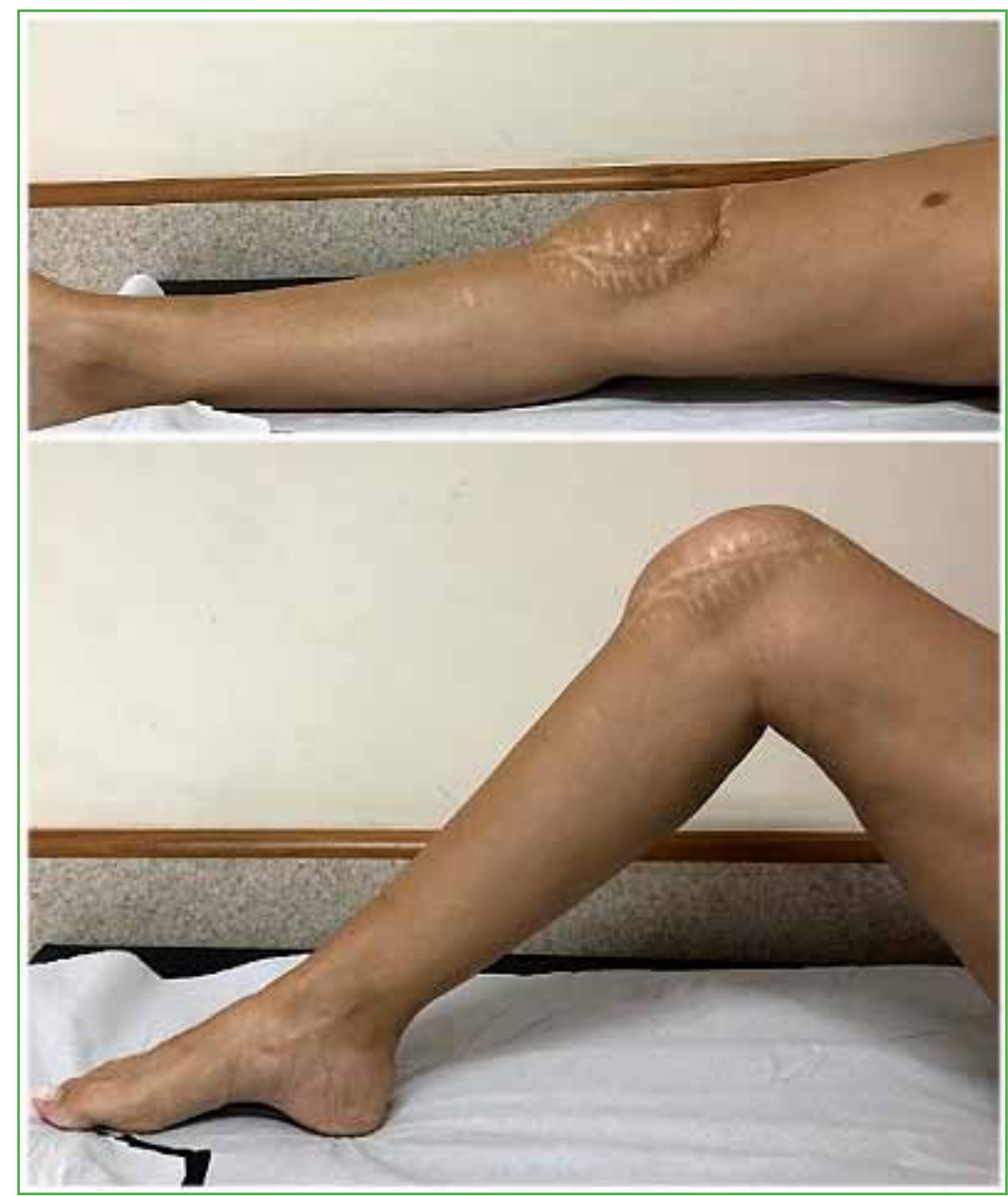

Figura 2. Imágenes clínicas del rango de movilidad posoperatorio. 
Las radiografías no revelan signos de aflojamiento (Figura 3). El KSS en la rodilla derecha mejoró a 86/90. La paciente refiere estar satisfecha con los resultados.

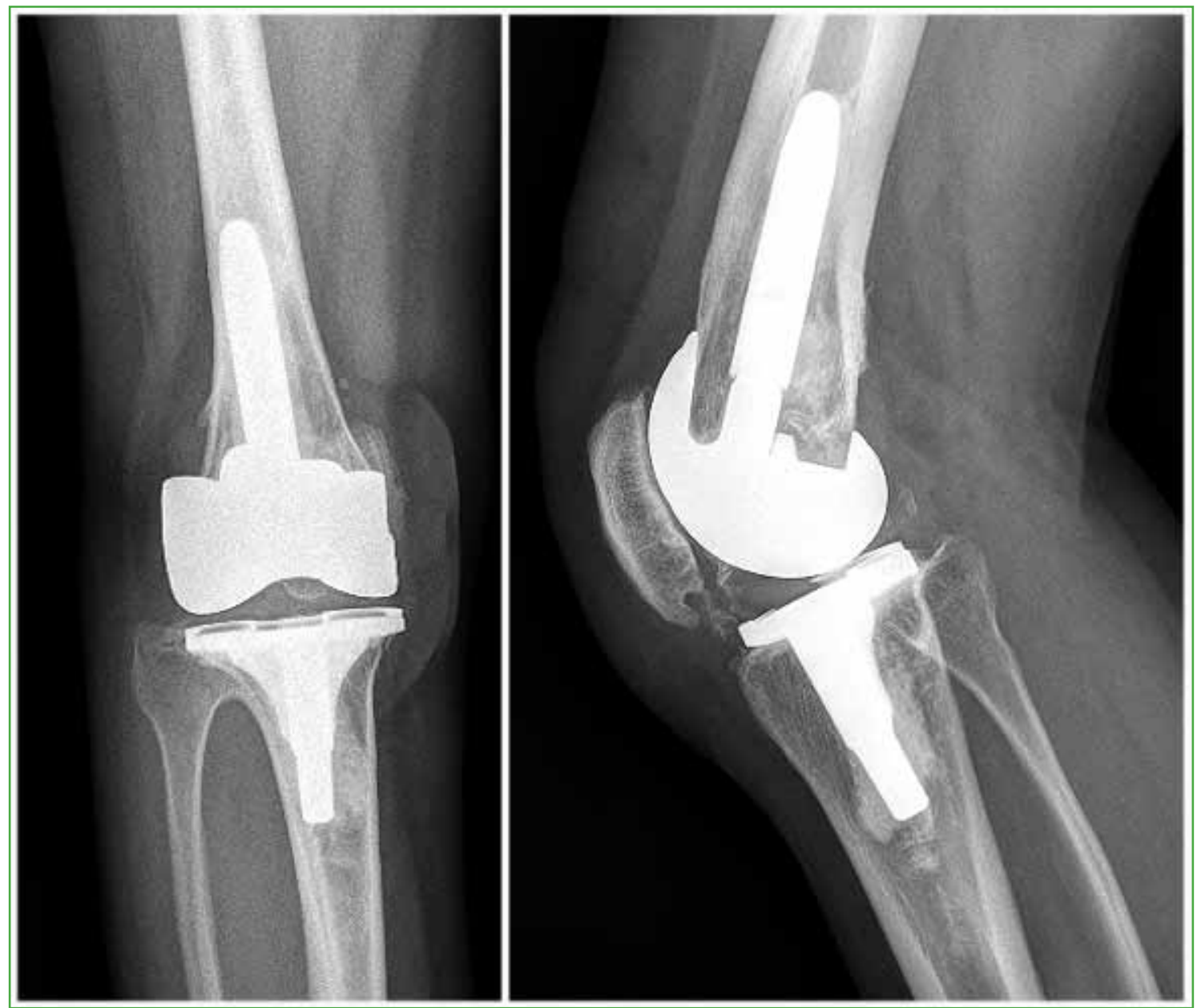

Figura 3. Radiografías posoperatorias de rodilla derecha, de frente y de perfil.

\section{Caso 2}

Hombre de 28 años, con antecedente de artritis reumatoide juvenil. Presenta anquilosis y valgo severo de ambas rodillas (Figura 4). En la rodilla izquierda, tiene una flexión de $70^{\circ}$ y, en la derecha, de $60^{\circ}$. El KSS era 11/45 para la rodilla derecha y 15/45 para la izquierda. Refiere severas limitaciones para realizar actividades de la vida diaria.

Se realizó un RTR bilateral en un solo acto quirúrgico (Figura 5). Se utilizó una prótesis estabilizada posterior con vástagos tibial y femoral para ambas rodillas. No tuvo complicaciones en el posoperatorio inmediato ni tardío. A los cuatro años de la cirugía, el rango de movilidad es de $100^{\circ}$ y la extensión de ambas rodillas es completa. El KSS mejoró notoriamente a 87/90 en la derecha y 80/90 en la izquierda. En las radiografías, no se observan signos de aflojamiento de la prótesis (Figura 6). Refiere estar satisfecho con el procedimiento. 


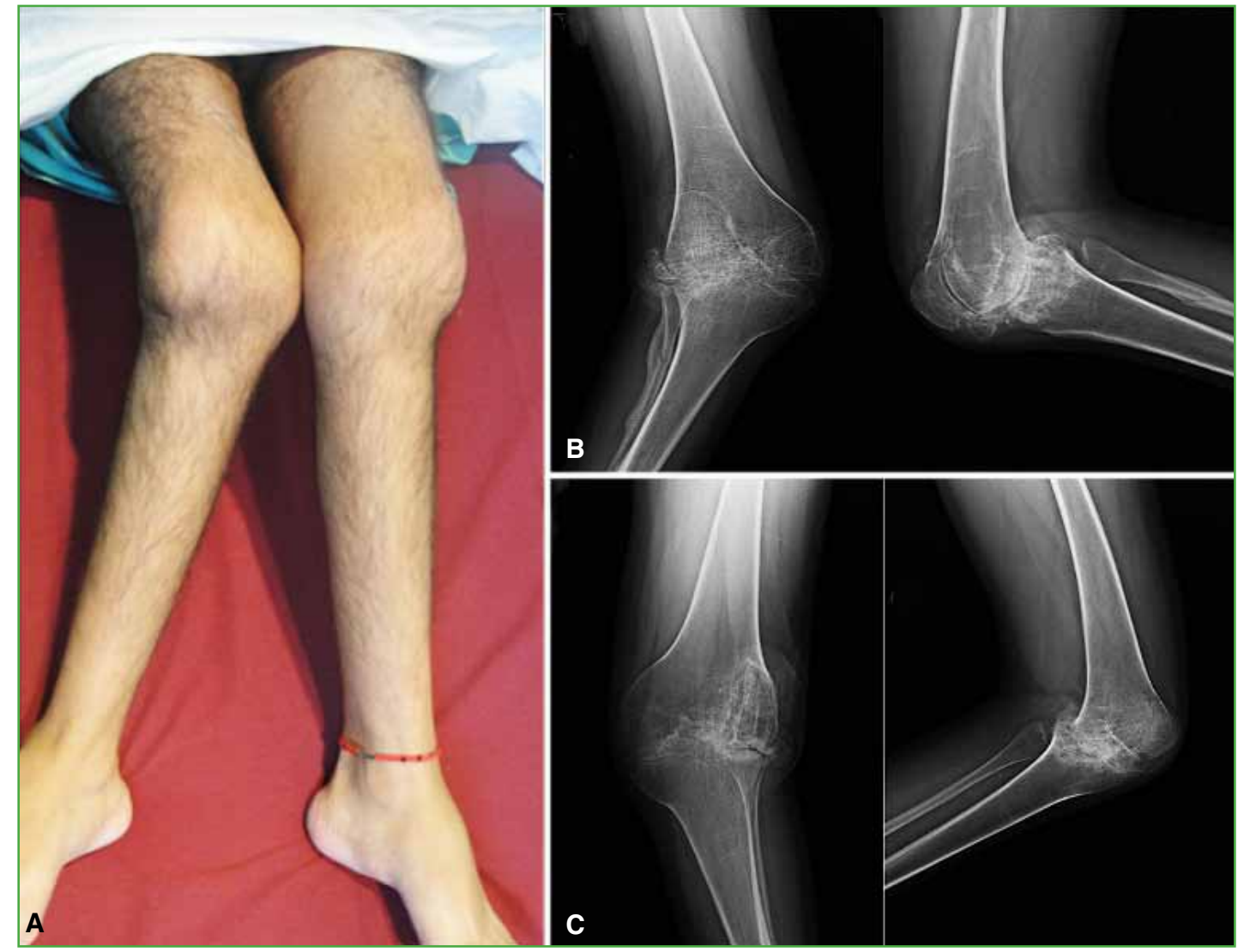

Figura 4. A. Imagen clínica de ambas rodillas que muestra la deformidad coronal y la anquilosis.

B y C. Radiografías preoperatorias de rodilla derecha e izquierda, de frente y de perfil.

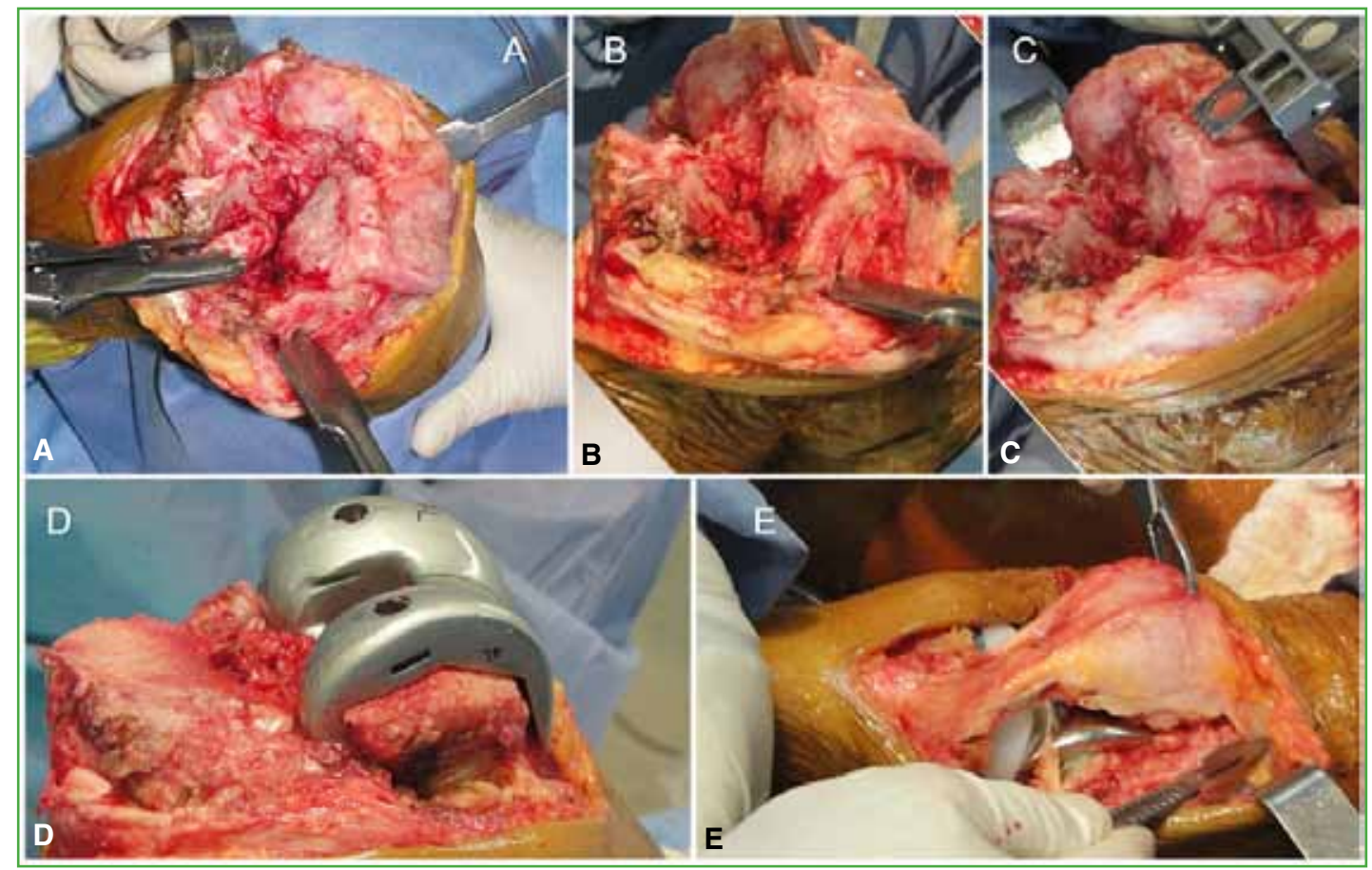

Figura 5. Imágenes intraoperatorias. A. Exposición del campo quirúrgico. B. Guía femoral endomedular.

C. Cortes óseos de fémur. D. Componente de prueba femoral. E. Liberación lateral de la rótula. 


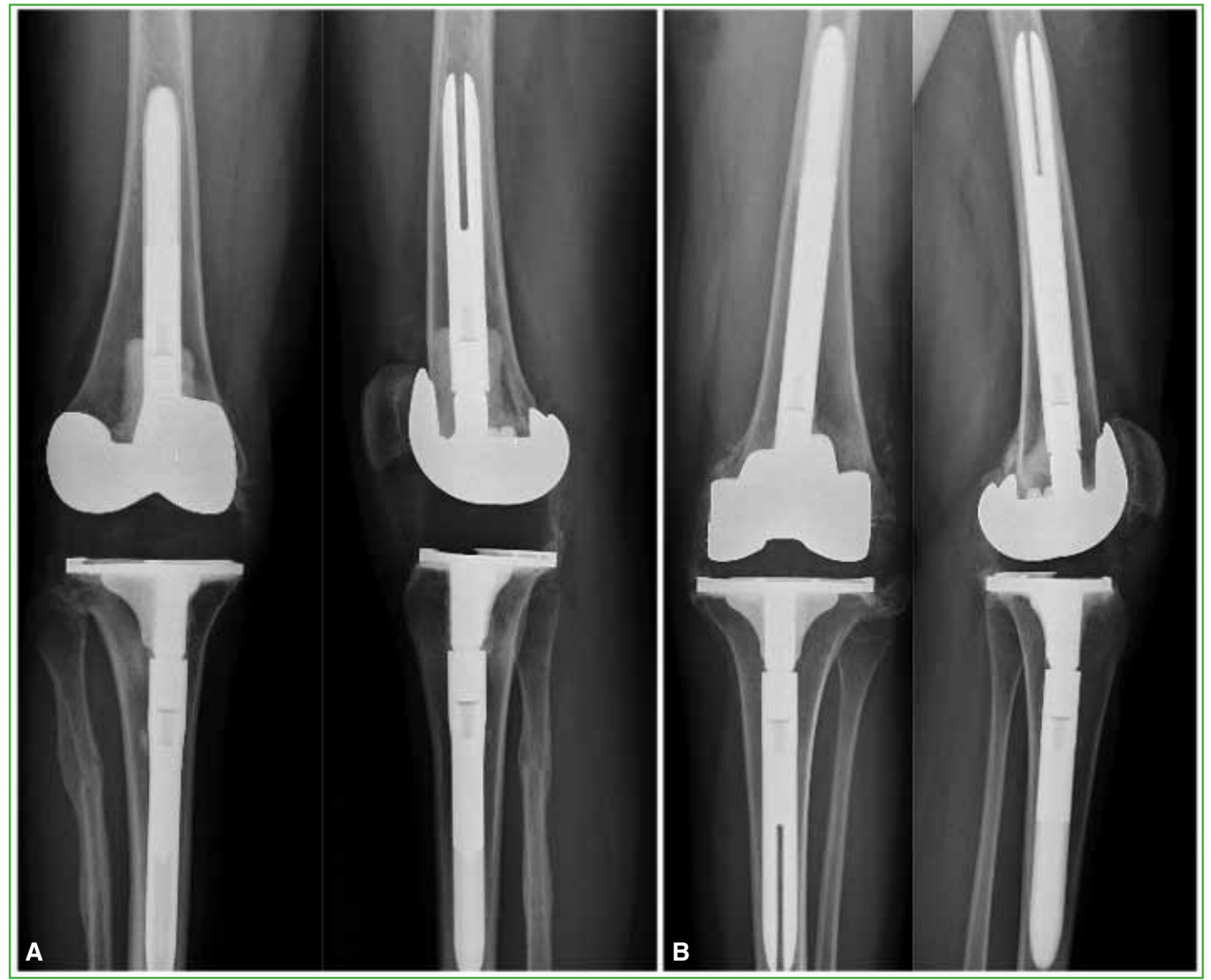

Figura 6. Radiografías posoperatorias de rodilla derecha (A) e izquierda (B), de frente y de perfil.

\section{Caso 3}

Hombre de 58 años con secuela de osteomielitis crónica en la rodilla izquierda. Presenta rodilla en extensión, sin movilidad y dolor. En la radiografía, se observa anquilosis rotulofemoral, sin unión ósea femorotibial, interpretada como anquilosis fibrosa (Figura 7A). El KSS preoperatorio era 43/55. No se pudo descartar una infección, por lo que se optó por un RTR en dos tiempos. Se utilizó para el reimplante una prótesis abisagrada rotatoria, debido a la incompetencia del complejo ligamentario medial. En el último control a los 18 meses de la cirugía, no había sufrido complicaciones, y tenía una flexión de $100^{\circ}$ y una extensión completa, con un KSS de 87/90 (Figura 7B).

\section{DISCUSIÓN}

El RTR en el contexto de una anquilosis o rigidez de rodilla representa un desafío para el cirujano, no solo por su alta demanda técnica, sino también por la elevada tasa de complicaciones posoperatorias. Presentamos una serie de tres pacientes, en la que las causas que llevaron a la anquilosis fueron la artritis reumatoide juvenil ( 2 casos) y una secuela infecciosa ( 1 caso). Estos pacientes fueron tratados de forma satisfactoria y no se produjeron complicaciones.

Es importante distinguir entre el concepto de rigidez y de anquilosis, muchas veces, confundidas como una única entidad, pero con claras diferencias en cuanto a la dificultad quirúrgica, las complicaciones y los resultados funcionales entre ambas. ${ }^{6.9}$ En los casos de rigidez, se hallan menores contracturas y adherencias de partes blandas, y la movilidad permite un mejor acceso, sobre todo, a la región posterior. Por lo tanto, el acceso a un apropiado campo quirúrgico y la obtención de las brechas en flexión y extensión se encuentran facilitados en comparación con los casos de anquilosis. 


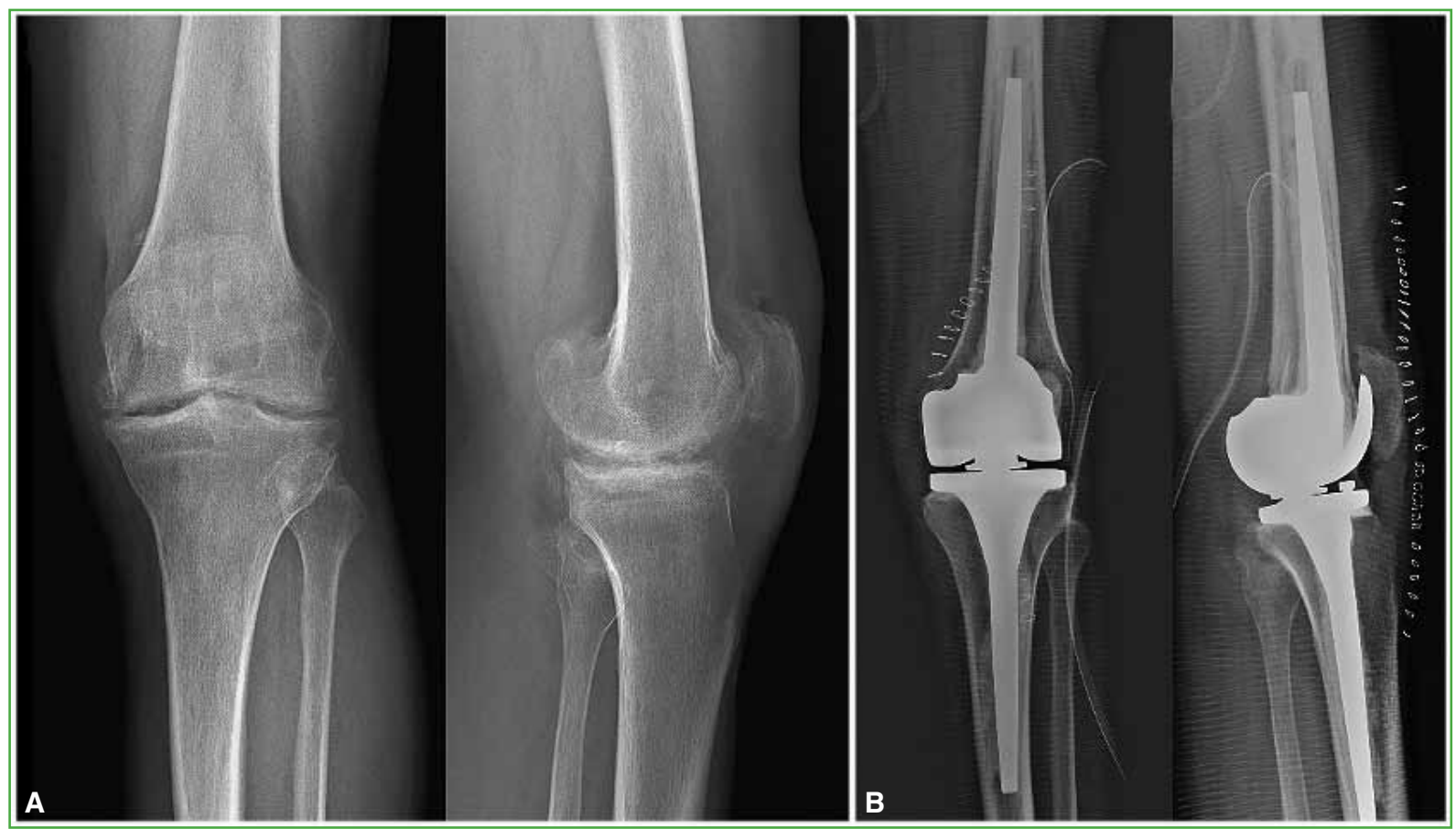

Figura 7. Radiografías de rodilla izquierda, de frente y de perfil, preoperatorias (A) y posoperatorias (B).

En la Tabla, se resumen las publicaciones sobre el tema de los últimos 30 años. Una de las series más extensas fue la de Kim y cols. publicada en 2008. ${ }^{20}$ Evaluaron una cohorte de 99 rodillas con anquilosis por un mínimo de seis años, y obtuvieron un incremento funcional promedio del Hospital for Special Surgery score de 60 a 81, del Western Ontario and McMaster Universities Osteoarthritis Index de 79 a 37 y del KSS de 53 a 85 . El rango de movilidad posoperatorio se incrementó entre $55^{\circ}$ y $105^{\circ}$, alcanzó un promedio de $79^{\circ}$. En los casos de Fosco y cols., y Polascik y cols. que comparan resultados en rodillas flexibles y rígidas, se reporta un mayor rango de movilidad posoperatorio en los pacientes con rodillas flexibles, pero el rango de movilidad adquirido en aquellos con rodillas rígidas se incrementó hasta $70^{\circ} .^{11,13}$ Esta diferencia se mantiene entre pacientes con rodillas rígidas y anquilóticas; Bae y cols. comunican una mejoría funcional en ambos grupos sin diferencia significativa, mientras que la mejoría del rango de movilidad es más notoria en el grupo con rigidez $\left(98,7^{\circ}\right.$ en el grupo con rigidez y $75,3^{\circ}$ en los pacientes con anquilosis).$^{6,9}$ Estos resultados fortalecen la idea enunciada previamente de que la movilidad preoperatoria condiciona la movilidad posoperatoria. ${ }^{3,4,12}$ Otro aspecto de interés es que, en los pacientes con anquilosis o rigidez en flexión, se logran mejores resultados de movilidad posoperatoria que en aquellos en extensión. ${ }^{6,12}$ La ganancia de rango de movilidad obtenida de $90^{\circ}$ a $100^{\circ}$ en nuestra serie coincide con la reportada, que es de $50^{\circ}-90^{\circ} .2,3,7,9,12,14,20,21$

A pesar de los buenos resultados publicados, la decisión quirúrgica debe ser tomada en conjunto con el paciente. Es de vital importancia conocer sus expectativas respecto del procedimiento por realizar y ser claros sobre los riesgos y beneficios. ${ }^{1}$ Aun así, los pacientes, en su mayoría, optan por operarse. ${ }^{13}$ Para conseguir una cirugía exitosa se debe realizar una correcta planificación prequirúrgica, haciendo foco en el estado de las partes blandas. El abordaje seleccionado debe ser el que cause menos agresión a las partes blandas, pero con una exposición cómoda. Si esto no se consigue, principalmente en rigideces en extensión, es necesario ampliar el campo para evitar complicaciones intraoperatorias. ${ }^{3,12}$ Kovalak y cols. prefieren la osteotomía de la tuberosidad anterior de la tibia, pero no hay que olvidar los riesgos de pérdida de fijación en pacientes osteoporóticos, la dificultad con el uso del vástago tibial y el riesgo de rotura del tendón. ${ }^{14}$ Aglietti y cols., y Kim y cols. realizan una cuadriceplastia en V-Y en todos los casos para permitir una mejor flexión y obtener un mayor campo quirúrgico, con buenos resultados al año tras una rehabilitación agresiva. ${ }^{8,10}$ 
Tabla. Revisión cronológica de la bibliografía

\begin{tabular}{|c|c|c|c|c|c|}
\hline Autor (Año) & $\begin{array}{l}\text { Cantidad de } \\
\text { rodillas }\end{array}$ & $\begin{array}{l}\text { Seguimiento } \\
\text { (rango) }\end{array}$ & Puntaje clínico & $\begin{array}{l}\text { Rango de movilidad } \\
\text { preoperatorio/posoperatorio }\end{array}$ & Complicaciones \\
\hline $\begin{array}{l}\text { Bradley y cols. }{ }^{2} \\
(1987)\end{array}$ & 9 rodillas & 3.6 años $(2-6)$ & & $0^{\circ} / 64^{\circ}\left(15^{\circ}-90^{\circ}\right)$ & $33,3 \%$ ( 3 casos $)$ \\
\hline $\begin{array}{l}\text { Aglietti y cols. }{ }^{10} \\
(1989)\end{array}$ & $\begin{array}{l}20 \text { rigidez } \\
6 \text { anquilosis }\end{array}$ & 4.5 años (2.5-7.5) & $\begin{array}{l}\text { HSS preop.: } 34(20-46) \\
\text { HSS posop.: } 74(0-94) \\
\text { No discrimina por grupo }\end{array}$ & $\begin{array}{l}\text { Rigidez } 32^{\circ} / 82^{\circ} \\
\text { Anquilosis } 0^{\circ} / 68^{\circ}\end{array}$ & $\begin{array}{l}7,5 \% \text { aflojamiento e } \\
\text { infección profunda }\end{array}$ \\
\hline $\begin{array}{l}\text { Naranjay cols. }{ }^{16} \\
(1996)\end{array}$ & $\begin{array}{l}6 \text { fusión } \\
31 \text { anquilosis }\end{array}$ & 7.5 años $(0.2-12)$ & & $0^{\circ} / 7^{\circ}-62^{\circ}$ & $53 \%$ \\
\hline $\begin{array}{l}\text { Kim y cols. } \\
(2000)\end{array}$ & $\begin{array}{l}14 \text { fusión } \\
16 \text { anquilosis }\end{array}$ & 5.3 años $(5-6)$ & $\begin{array}{l}\text { Fusión } \\
\text { KSS preop.: } 60, \text { KSS posop.: } 73 \\
\text { Anquilosis } \\
\text { KSS preop.: } 60, \text { KSS posop.: } 73\end{array}$ & $\begin{array}{l}\text { Anquilosis } \\
0^{\circ} / 75,7^{\circ}\left(70^{\circ}-95^{\circ}\right) \\
\text { Fusión } \\
0^{\circ} / 75,9^{\circ}\left(70^{\circ}-95^{\circ}\right)\end{array}$ & $\begin{array}{l}53,3 \% \text { necrosis de la } \\
\text { herida }\end{array}$ \\
\hline $\begin{array}{l}\text { McAuleyy cols. }{ }^{21} \\
(2002)\end{array}$ & 27 rodillas & 6 años $(2.3-11.8)$ & & $30^{\circ}\left(0^{\circ}-50^{\circ}\right) / 74^{\circ}\left(15^{\circ}-110^{\circ}\right)$ & $41 \%$ \\
\hline $\begin{array}{l}\text { Rajgopal y cols. }{ }^{19} \\
(2005)\end{array}$ & 84 rodillas & 9 años (4-15) & $\begin{array}{l}\text { KSS preop.: } 14,6 / 26,8 \\
\text { KSS posop.: } 75 / 84\end{array}$ & $<20^{\circ} / 75^{\circ}\left(55^{\circ}-100^{\circ}\right)$ & $7,36 \%$ \\
\hline Bae y cols. ${ }^{9}(2005)$ & $\begin{array}{l}12 \text { rigidez } \\
20 \text { anquilosis }\end{array}$ & 10 años (5-13) & $\begin{array}{l}\text { Rigidez } \\
\text { HSS preop.: 52,1 (29-66) } \\
\text { HSS posop.: 86,7 (69-97) } \\
\text { Anquilosis } \\
\text { HSS preop.: 59,7 (26-87) } \\
\text { HSS posop.: 84,9 (64-94) }\end{array}$ & $\begin{array}{l}\text { Rigidez } 49,5^{\circ}\left(15^{\circ}-80^{\circ}\right) / 98,7^{\circ}\left(60-130^{\circ}\right) \\
\text { Anquilosis } 0^{\circ} / 75,3^{\circ}\left(30^{\circ}-115^{\circ}\right)\end{array}$ & $12,5 \%$ \\
\hline Bhan y cols. ${ }^{6}(2006)$ & $\begin{array}{l}64 \text { rigidez } \\
26 \text { anquilosis }\end{array}$ & 6.5 años (2-10) & $\begin{array}{l}\text { Rigidez } \\
\text { KSS preop.: } 34,5 \\
\text { KSS posop.: } 89,2 \\
\text { Anquilosis } \\
\text { KSS preop.: } 47 \\
\text { KSS posop.: } 75\end{array}$ & $\begin{array}{l}\text { Rígidez } 35^{\circ}-69^{\circ} / 1^{\circ}-94^{\circ} \\
\text { Anquilosis } 0^{\circ} / 3^{\circ}-74^{\circ}\end{array}$ & $\begin{array}{l}33 \% \text { generales } \\
50 \% \text { de necrosis de la } \\
\text { herida en el grupo de } \\
\text { anquilosis vs. } 7 \% \text { en el } \\
\text { grupo de rigidez }\end{array}$ \\
\hline $\operatorname{Kim} y \operatorname{Kim}^{20}(2008)$ & 99 rodillas & 8.9 años (6.6-14) & $\begin{array}{l}\text { HSS preop.: } 60 \\
\text { HSS posop.: } 81 \\
\text { WOMAC preop.: } 79 \\
\text { WOMAC posop.: } 37 \\
\text { KSS preop.: } 53 \\
\text { KSS posop.: } 85\end{array}$ & $0^{\circ} / 79^{\circ}\left(55^{\circ}-115^{\circ}\right)$ & $\begin{array}{l}27 \% \\
17 \% \text { necrosis de la } \\
\text { herida }\end{array}$ \\
\hline Camanho $^{15}(2009)$ & 9 rodillas & 5 años & $\begin{array}{l}\text { HSS preop.: } 55,9 \\
\text { HSS posop.: } 81,8\end{array}$ & $<5^{\circ} / 65^{\circ}\left(0^{\circ}-90^{\circ}\right)$ & 2 casos \\
\hline $\begin{array}{l}\text { Kim y } \mathrm{Kim}^{7} \\
(2009)\end{array}$ & 86 rodillas & 9.1 años $(5-12)$ & $\begin{array}{l}\text { KSS preop.: } 11 \\
\text { KSS posop.: } 90 \\
\text { WOMAC preop.: } 79 \text { (78-96) } \\
\text { WOMAC posop.: } 34,4(5-68) \\
\text { HSS preop.: } 42 \\
\text { HSS posop.: } 84\end{array}$ & $40^{\circ}\left(10^{\circ}-50^{\circ}\right) / 102^{\circ}\left(65^{\circ}-145^{\circ}\right)$ & $\begin{array}{l}14 \% \\
6 \% \text { necrosis de la herida }\end{array}$ \\
\hline $\begin{array}{l}\text { Fosco y cols. }{ }^{11} \\
(2011)\end{array}$ & $\begin{array}{l}32 \text { rodillas casos } \\
28 \text { rodillas } \\
\text { controles }\end{array}$ & 2 años & $\begin{array}{l}\text { Casos } \\
\text { KSS preop.: } 31,3 / 34 \\
\text { KSS posop.: } 85,2 / 70,8 \\
\text { Controles } \\
\text { KSS preop.: } 29,3 / 37,8 \\
\text { KSS posop.: } 92,6 / 84\end{array}$ & $\begin{array}{l}\text { Casos } 29^{\circ} / 87^{\circ} \\
\text { Controles } 86^{\circ} / 106^{\circ}\end{array}$ & $\begin{array}{l}\text { Casos } 21,8 \% \\
\text { Controles } 6,2 \%\end{array}$ \\
\hline Hsu y cols. ${ }^{3}(2012)$ & 39 rodillas & 4.8 años $(2-10.25)$ & $\begin{array}{l}\text { KSS preop.: } 33 / 11 \\
\text { KSS posop.: 80/41 } \\
\text { WOMAC preop.: } 71 \\
\text { WOMAC posop.: } 35\end{array}$ & $35^{\circ}\left(0^{\circ}-50^{\circ}\right) / 94^{\circ}\left(0^{\circ}-135^{\circ}\right)$ & $\begin{array}{l}43,6 \% \\
\text { necrosis de la herida } \\
7,7 \%\end{array}$ \\
\hline $\begin{array}{l}\text { Kovalak y cols. }{ }^{14} \\
(2015)\end{array}$ & 6 rodillas & 7.1 años (1.8-10.5) & $\begin{array}{l}\text { HSS preop.: } 19,5 \\
\text { HSS posop.: } 57,5 \\
\text { WOMAC preop.: } 39,75 \\
\text { WOMAC posop.: } 62,4\end{array}$ & $0-20^{\circ} / 85^{\circ}\left(75^{\circ}-95^{\circ}\right)$ & 2 casos \\
\hline $\operatorname{Kim}^{17}(2017)$ & 10 rodillas & 10.2 años (1-19.5) & $\begin{array}{l}\text { KSS preop.: } 42,6 / 39 \\
\text { KSS posop.: } 68,6 / 66\end{array}$ & $9,5^{\circ}\left(0^{\circ}-30^{\circ}\right) / 8,5^{\circ}\left(15^{\circ}-115^{\circ}\right)$ & $\begin{array}{l}3 \text { casos } \\
1 \text { necrosis de la herida }\end{array}$ \\
\hline $\begin{array}{l}\text { Rajgopaly cols. }{ }^{12} \\
(2017)\end{array}$ & $\begin{array}{l}51 \text { rodillas } \\
\text { extensión } \\
64 \text { rodillas flexión }\end{array}$ & 9.7 años (2-17.5) & $\begin{array}{l}\text { Extensión preop. KSS: } 23,2 / 14,4 \\
\text { Extensión posop. KSS: } 74,1 / 66,4 \\
\text { Flexión preop. KSS: } 14 / 8,3 \\
\text { Flexión posop. KSS: } 71,2 / 64,3\end{array}$ & $\begin{array}{l}\text { Extensión } \\
10,9^{\circ}\left(0^{\circ}-20^{\circ}\right) / 86,5^{\circ}\left(30^{\circ}-120^{\circ}\right) \\
\text { Flexión } \\
8,7^{\circ}\left(<20^{\circ}\right) / 92,2^{\circ}\left(45^{\circ}-110^{\circ}\right)\end{array}$ & $\begin{array}{l}\text { Extensión } 23,5 \% \\
\text { Flexión } 18,75 \%\end{array}$ \\
\hline $\begin{array}{l}\text { Polasciky cols. }{ }^{13} \\
\text { (2018) }\end{array}$ & $\begin{array}{l}28 \text { rodillas rígidas } \\
56 \text { rodillas } \\
\text { flexibles }\end{array}$ & 4.3 años $(2.5-6.3)$ & $\begin{array}{l}\text { Casos preop. KSS: } 28 / 42 \text { - OKQS: } 39 \\
\text { Casos posop. KSS: } 78 / 64-\text { OKQS: } 22 \\
\text { Controles preop. KSS: } 35 / 49 \text { - OKQS: } 39 \\
\text { Controles posop. KSS: } 83 / 75 \text { - OKQS: } 19\end{array}$ & $\begin{array}{l}\text { Casos } 8^{\circ}\left( \pm 7^{\circ}\right) / 80^{\circ}\left( \pm 33^{\circ}\right) \\
\text { Controles } 116^{\circ}\left( \pm 17^{\circ}\right) / 118^{\circ}\left( \pm 12^{\circ}\right)\end{array}$ & $\begin{array}{l}\text { Casos } 10 \% \text { revisiones } \\
\text { Controles } 0 \% \text { revisiones }\end{array}$ \\
\hline $\begin{array}{l}\text { Bittelmany cols. }{ }^{18} \\
(2019)\end{array}$ & 39 rodillas & 4-16 años & $\begin{array}{l}\text { HSS preop.: } 30 \\
\text { HSS posop.: } 77\end{array}$ & $<30^{\circ} / 80^{\circ}$ & $20,5 \%$ \\
\hline
\end{tabular}

Preop. $=$ preoperatorio, posop. $=$ posoperatorio, HSS $=$ Hospital for Special Surgery score, WOMAC $=$ Western Ontario and McMaster $($ WOMAC) Universities Osteoarthritis Index, OKQS = Oxford Knee Questionnaire Score. 
Posteriormente, en 2009, Kim y cols. reservan la cuadriceplastia para menos del 50\% de los casos; Bae y cols. la utilizaron en el $30 \%$ de sus pacientes, y obtuvieron un resultado inferior que con la osteotomía de la tuberosidad anterior de la tibia. ${ }^{7,9}$ Agregar este gesto quirúrgico puede generar un déficit de extensión de hasta $20^{\circ}$, por lo que creemos que debe ser usado cautelosamente. ${ }^{12}$ Fosco y cols., y Polascik y cols. prefieren realizar una liberación lateral en todos los casos, agregando una osteotomía de la tuberosidad anterior de la tibia o rectus snip, según corresponda. ${ }^{11,13}$

La regla que rige la elección del diseño de la prótesis es la menor constricción que genere una suficiente estabilidad. De esta forma, evitamos una innecesaria constricción que incrementa el estrés en la interfase hueso-cemento, acelerando el aflojamiento de la prótesis. ${ }^{8}$ Decidimos usar un diseño abisagrado rotatorio en el paciente con una secuela de infección, ya que los sucesivos desbridamientos comprometieron las estructuras ligamentarias y, en los otros dos casos, se optó por un diseño de estabilización posterior con vástago. Esta selección depende mucho de las preferencias del cirujano, como Bae y cols. quienes colocaron, en todos los pacientes, diseños de retención del cruzado posterior o de estabilización posterior, sin tener revisiones por aflojamiento a los 10 años. ${ }^{9}$ Kim y cols. comunican que prueban el diseño de estabilización posterior durante la cirugía, si consigue una estabilidad coronal lo seleccionan; de lo contrario, realizan pruebas con el diseño de constricción condilar que, en el resto de los casos, fueron satisfactorios y no informan revisiones por aflojamiento durante un plazo mínimo de cinco años. ${ }^{7}$ Bhan y cols., y Rajgopal y cols. lograron una supervivencia de la prótesis del $100 \%$ en todos los casos, durante un período de entre 6 y 15 años, tratados con diseño de estabilización posterior y constricción condilar. ${ }^{6,19}$ En otros casos, las fallas fueron más evidentes, Polascik y cols. reportaron el uso de diseños de estabilización posterior en 28 rodillas rígidas, con una tasa de revisión del $10 \%$ a los 5 años. ${ }^{13}$

Las características quirúrgicas y de los pacientes hacen esta cirugía muy riesgosa desde la perspectiva de las complicaciones. En nuestros casos, no hemos tenido complicaciones tempranas ni tardías. Pero, como ya hemos mencionado, la forma de prevenir las complicaciones es con una apropiada planificación. La necrosis de la herida es la complicación más frecuente, tiene una incidencia alta, hasta del $50 \% \cdot{ }^{6,8,20}$ Esto se debe a múltiples causas, por un lado, las cirugías previas que puedan haber alterado la irrigación cutánea y provocado adherencias que limiten la elasticidad de la piel. Además, la combinación del aumento del contenido, por el tamaño de la prótesis, y la movilidad ganada, van a generar una tensión continua a través de la rótula llevando al sufrimiento cutáneo. La expansión cutánea con balón antes de la cirugía es una forma de prevenirlo, mediante la distensión controlada de los tejidos blandos de la zona. ${ }^{22}$ Además, se puede evitar mediante rotuloplastia o restringiendo la movilidad posoperatoria, afrontando el riesgo de una movilidad posoperatoria inferior. ${ }^{6,820}$ Otra complicación no despreciable es el aflojamiento, explicado por la necesidad de implantes constreñidos en pacientes jóvenes con buena funcionalidad y la dificultad técnica generada por la distorsión de la anatomía que presentan, que llevan a una incorrecta colocación de los componentes que aceleran el aflojamiento.$^{13}$ Por el contrario, algunos estudios reportan tasas bajas de revisiones, similares a las publicadas en rodillas flexibles, que varían del $0 \%$ al $4 \%$ en seguimientos $>9$ años., ${ }^{3,220}$ La tasa de revisión por infección o fractura alrededor de la prótesis es $<10 \%$ según lo publicado., ${ }^{3,11,12}$

A pesar de ser un reporte de tres casos, creemos que el RTR en pacientes con anquilosis es una opción terapéutica valiosa. Esto se debe principalmente a que se trata, en su mayoría, de adultos jóvenes y tiene un alto impacto en la calidad de vida. Para optimizar el éxito quirúrgico debe estar a cargo de un equipo entrenado y es necesaria una meticulosa planificación prequirúrgica. De todos modos, el paciente debe conocer el mayor riesgo de complicaciones de esta intervención, pero aun así obtuvimos una alta tasa de satisfacción.

Conflicto de intereses: Los autores no declaran conflictos de interés.

ORCID de I. García-Mansilla: https://orcid. org/0000-0002-7247-3734 ORCID de T. I. Nicolino: https://orcid. org/0000-0002-9550-3713 ORCID de C. Yacuzzi: https://orcid. org/0000-0002-7732-7883
ORCID de L. Carbó: https://orcid.org/0000-0002-8053-0890

ORCID de J. Costantini: https://orcid.org/0000-0001-8900-6254 ORCID de M. Costa Paz: https://orcid. org/0000-0002-8217-1086 


\section{BIBLIOGRAFÍA}

1. Lange T, Schmitt J, Kopkow C, Rataj E, Günther K-P, Lützner J. What do patients expect from total knee arthroplasty? A Delphi Consensus Study on Patient Treatment Goals. J Arthroplasty 2017; 32:2093-9.e1. https://doi.org/10.1016/j.arth.2017.01.053

2. Bradley GW, Freeman MA, Albrektsson BE. Total prosthetic replacement of ankylosed knees. J Arthroplasty 1987;2(3):179-83. https://doi.org/10.1016/s0883-5403(87)80035-9

3. Hsu CH, Lin PC, Chen WS, Wang JW. Total knee arthroplasty in patients with stiff knees. J Arthroplasty 2012;27(2):286-92. https://doi.org/10.1016/j.arth.2011.05.001

4. Gatha NM, Clarke HD, Fuchs R, Scuderi GR, Insall JN. Factors affecting postoperative range of motion after total knee arthroplasty. J Knee Surg 2004;17(4):196-202. https://doi.org/10.1055/s-0030-1248221

5. Harvey IA, Barry K, Kirby SP, Johnson R, Elloy MA. Factors affecting the range of movement of total knee arthroplasty. J Bone Joint Surg Br 1993;75(6):950-5. https://doi.org/10.1302/0301-620x.75b6.8245090

6. Bhan S, Malhotra R, Kiran EK. Comparison of total knee arthroplasty in stiff and ankylosed knees. Clin Orthop Relat Res 2006;451:87-95. https://doi.org/10.1097/01.blo.0000229313.20760.13

7. Kim Y-H, Kim J-S. Does TKA improve functional outcome and range of motion in patients with stiff knees? Clin Orthop Relat Res 2009;467(5):1348-54. https://doi.org/10.1007/s11999-008-0445-7

8. Kim YH, Kim JS, Cho SH. Total knee arthroplasty after spontaneous osseous ankylosis and takedown of formal knee fusion. J Arthroplasty 2000;15(4):453-60. https://doi.org/10.1054/arth.2000.4640

9. Bae DK, Yoon KH, Kim HS, Song SJ. Total knee arthroplasty in stiff knees after previous infection. J Bone Joint Surg Br 2005;87(3):333-6. https://doi.org/10.1302/0301-620x.87b3.15457

10. Aglietti P, Windsor RE, Buzzi R, Insall JN. Arthroplasty for the stiff or ankylosed knee. J Arthroplasty 1989;4(1):15. https://doi.org/10.1016/s0883-5403(89)80046-4

11. Fosco M, Filanti M, Amendola L, Savarino LM, Tigani D. Total knee arthroplasty in stiff knee compared with flexible knees. Musculoesquelet Surg 2011;95(1):7-12. https://doi.org/10.1007/s12306-011-0099-6

12. Rajgopal A, Panda I, Dahiya V. A Comparative study on the long-term outcome of total knee arthroplasty performed for knees stiff in extension and those stiff in flexion. J Arthroplasty 2017;32(11):3396-403. https://doi.org/10.1016/j.arth.2017.06.027

13. Polascik BW, Bin Abd Razak HR, Chong H-C, Lo N-N, Yeo S-J. Acceptable functional outcomes and patient satisfaction following total knee arthroplasty in asians with severe knee stiffness: A matched analysis. Clin Orthop Surg 2018;10(3):337-43. https://doi.org/10.4055/cios.2018.10.3.337

14. Kovalak E, Can A, Stegemann N, Erdoğan AÖ, Erdoğan F. Total knee arthroplasty after osseous ankylosis of the knee joint. Acta Orthop Traumatol Turc 2015;49(5):503-7. https://doi.org/10.3944/AOTT.2015.14.0304

15. Camanho GL. Total arthroplasty in ankylosed knees: a case series. Clinics 2009;64(3):183-7. https://doi.org/10.1590/s1807-59322009000300006

16. Naranja RJ Jr, Lotke PA, Pagnano MW, Hanssen AD. Total knee arthroplasty in a previously ankylosed or arthrodesed knee. Clin Orthop Relat Res 1996;(331):234-7. https//doi.org/10.1097/00003086-199610000-00033

17. Kim H-J, Mun J-U, Kim K-H, Kyung H-S. Total knee arthroplasty conversion for patients with ankylosed knees. J Orthop Surg 2017;25(1):2309499016684095. https://doi.org/10.1177/2309499016684095

18. Bittelman S, Nemtala F, Salvo N, Araya R, Devia B, Voss C, et al. Prótesis de rodilla en pacientes con anquilosis. Revista Acaro 2019;5(2):31-4. Disponible en: http://acaro.org.ar/acarorevista/images/revistas/05_02/AC_05_02_v6.pdf

19. Rajgopal A, Ahuja N, Dolai B. Total knee arthroplasty in stiff and ankylosed knees. J Arthroplasty 2005;20(5):58590. https://doi.org/10.1016/j.arth.2005.04.002

20. Kim Y-H, Kim J-S. Total knee replacement for patients with ankylosed knees. J Bone Joint Surg Br 2008;90(10): 1311-6. https://doi.org/10.1302/0301-620X.90B10.20857

21. McAuley JP, Harrer MF, Ammeen D, Engh GA. Outcome of knee arthroplasty in patients with poor preoperative range of motion. Clin Orthop Relat Res 2002;404:203-7. https://doi.org/10.1097/00003086-200211000-00033

22. Cho SH, Jeong ST, Park HB, Hwang SC, Kim DH. Two-stage conversion of fused knee to total knee arthroplasty. $J$ Arthroplasty 2008;23(3):476-9. https://doi.org/10.1016/j.arth.2007.06.013 